\title{
Adherence to the United States Preventative Services Task Force Female Screening Guidelines: An Internal Medicine Residency Program Experience
}

Chikezie K. Alvarez ${ }^{1}$, Julvet Mbianda ${ }^{2}$, Muhammad Nadeem ${ }^{3}$, Shahryar Anwar Ansari ${ }^{2}$, Ahmed Dirweesh $^{2}$, Mohammad Arsalan ${ }^{3}$, Shaikh Fawwad ${ }^{2}$, Vincent A. DeBari ${ }^{2}$, Donald Christmas ${ }^{3}$, Sara L. Wallach ${ }^{3}$

1. Medicine, St. Francis Medical Center, Trenton, USA 2. Internal Medicine, St. Francis Medical Center, Trenton, USA 3. Internal Medicine, Hackensack Meridian School of Medicine at Seton Hall University, Nutley, USA

$\square$ Corresponding author: Chikezie K. Alvarez, chikeziealvarez@gmail.com Disclosures can be found in Additional Information at the end of the article

\section{Abstract}

\section{Background}

Various guidelines exist for female preventative screening tests and medical resident physician adherence to the United States Preventive Services Task Force (USPSTF) guidelines varies. National screening rates for breast cancer and osteoporosis have improved but they are still below the expected target.

\section{Material and methods}

Ambulatory medical clinic records of female patients from the period July 2015 to December 2017 were reviewed for breast cancer and osteoporosis screening. Resident performance and commitment with regards to ordering the aforementioned screening tests according to the USPSTF guidelines were compared to the most recent national screening rates for mammograms and dualenergy X-ray absorptiometry (DXA) scans.

\section{Results}

Of the 1327 charts reviewed, 1025 was included in the study. Of the 545 mammograms performed, 93\% of them were indicated according to the USPSTF guidelines ( $\mathrm{P}<0.0001,95 \%$ CI: 125.9-342.0). A total of 480 mammograms were not ordered, of which $6 \%$ were indicated and $93.9 \%$ were not indicated. Out of a total of 107 DXA scans performed, $88.7 \%$ were correctly indicated $(\mathrm{P}<0.0001$, 95\% CI: 37.11-132.9).

\section{Conclusion}

Resident physician adherence to the USPSTF screening guidelines for breast cancer and DXA scans were higher than the national and state screening rates. Our well-structured educational project (strong faculty mentorship, resident to patient continuity of care and the reasonable residentclinic load) resulted in higher screening rates.

Categories: Internal Medicine, Medical Education

Keywords: mammogram, dxa scan, united states preventive services task force, breast cancer screening, osteoporosis screening 


\section{Introduction}

In 2016, United States Preventive Services Task Force (USPSTF) issued an update to their 2009 breast cancer screening recommendations. The changes were subtle regarding women aged 50-74 [1]. Their most recent updates to osteoporosis and cervical cancer screening were published in 2011 and 2012, respectively [2,3]. Various societies and organizations have similar but sometimes varying degrees of screening guideline parameters for breast cancer and osteoporosis prevention. Multiple studies have shown varying degrees of physicians' adherence to cancer screening guidelines issued by USPSTF [4-7]. They also showed that residents tend to have a low performance with prevention screening. This performance improves with experience [5]. As a teaching institution, we performed a chart review to assess our residents' adherence to preventive testing to look for training improvement opportunities. Our aim was to assess our resident physician performance and adherence to the recommended screening guidelines for breast cancer and osteoporosis according to the USPSTF guidelines. We also compared our screening rates to the national screening rates for breast cancer and osteoporosis. We share our findings to provide an insight into a community-based teaching institutions' experience and also to shed some light into the challenges that face similar programs.

\section{Materials And Methods}

The study was approved by the Institutional Review Board (IRB). This was a cross-sectional study which retrospectively reviewed ambulatory medical clinic female records $(n=1025)$ from the period July 2015 to December 2017 at an urban teaching healthcare facility in New Jersey, USA and it was approved by the IRB. Charts were reviewed to assess resident physician adherence to the USPSTF screening guidelines for cervical cancer and osteoporosis via screening mammography, and dual-energy X-ray absorptiometry (DXA) scans.

Resident performance and commitment with regards to ordering the aforementioned screening tests when applicable according to the USPSTF guidelines were compared to the most recent national screening rates for mammograms and DXA scans [8-10]. This quality improvement project utilized this time period so that an assessment can be made of the current PGY-1, PGY-2 and PGY3 internal medicine residents class. We included individuals who completed DXA and mammogram testing, as confirmed by the hospitals' electronic medical records (EMR). Charts were also reviewed to assess residence adherence to pap testing; however we chose not to include it in our analysis as we could not verify if they were completed by patients. Patients performed pap tests at an external outpatient office and the confirmatory results were not available to us via our EMR. As a result, we could not directly confirm whether pap tests were performed. We sought to assess whether the appropriate screening tests were ordered and performed if indicated, as well as if testing was done even when it was not appropriately indicated. Total of 1,327 charts were reviewed, of which 1,025 charts were selected for inclusion in our study after meeting the predefined set inclusion and exclusion criteria.

\section{Inclusion criteria}

Females >21 years of age who presented to our ambulatory clinic from July 2015 to December 2017 and were seen by medical residents. We included females whose mammogram and osteoporosis screening test were documented in our hospitals' clinic EMR records.

\section{Exclusion criteria}

Females <21 years of age, inpatient population, all males, women with a family history of breast cancer, personal history of breast cancer, known BRCA1/BRCA2 mutations or a history of chest radiation between ages 10 and 30 years (for breast cancer screening). Corticosteroid use $>3$ months, family history of osteoporosis, previous fracture, women $<65$ years with a FRAX score $>9.3 \%$, parental history of hip fracture, greater than two drinks alcohol/day, rheumatoid arthritis, current BMI < 20 (for osteoporosis screening). Charts of patients who were not seen by residents were excluded. Charts of patients who did not have documented proof of completed mammograms 


\section{Cureus}

and DXA scans were excluded.

Results were computed into three separate 2 x 2 contingency tables for screening mammography, and DXA scans. Descriptive statistics with cross-tabulations were performed. The Fisher exact test was used to examine the association between variables. Odds ratio was used as a measure of effect size. Comparisons which yielded P-values less than 0.05 on a two-sided basis were considered statistically significant.

\section{Results}

Of the 545 mammograms ordered and completed, 93\% (507 of 545) of them were indicated according to the USPSTF guidelines and $6.97 \%$ (38 of 545) were not indicated. A total of 480 mammograms were not ordered, of which 6\% (29 of 480) were indicated and 93.9\% (451 of 480) were not indicated $(\mathrm{P}<0.0001)$ (Table 1$)$.

\begin{tabular}{|c|c|c|c|}
\hline & Mammogram Performed & Mammogram Not Performed & Total \\
\hline Mammogram Indicated & 507 & 29 & 536 \\
\hline Mammogram Not Indicated & 38 & 451 & 489 \\
\hline Iotal & 545 & 480 & 1025 \\
\hline \multicolumn{4}{|c|}{$\mathrm{P}<0.0001$ (95\% Cl: 125.9-342.0). Odds ratio: 207.5} \\
\hline
\end{tabular}

\section{TABLE 1: Mammography Results}

Out of a total of 107 DXA scans ordered and completed, 88.7\% (95 of 107) were correctly indicated and 11.2\% (12 of 107) were not $(\mathrm{P}<0.0001)$. A total of 918 DXA scans were not ordered, of which 10.1\% (93 out of 918) DXA scans were indicated according to the USPSTF guidelines and $89.8 \%$ (825 out of 918) DXA scans not indicated $(\mathrm{P}<0.0001)$ (Table 2).

\begin{tabular}{|c|c|c|c|}
\hline & DXA Performed & DXA Not Performed & Total \\
\hline DXA Indicated & 95 & 93 & 188 \\
\hline DXA Scan not Indicated & 12 & 825 & 837 \\
\hline Total & 107 & 918 & 1025 \\
\hline
\end{tabular}

\section{TABLE 2: DXA Results}

DXA: Dual-energy X-ray absorptiometry; Cl: Confidence interval.

\section{Discussion}

The aim of this study was to assess our institutions' resident physician adherence to the USPSTF screening guidelines with regards to mammograms and DXA scans. The demographic population 
of our medical clinic consists of 40\% Hispanic, 30\% Black, and 30\% “Others”. We also serve and treat a significant indigent population with our ambulatory medical clinic consisting of $50 \%$ uninsured individuals on charity care, $40 \%$ on managed Medicaid and 10\% on Medicare.

The burden of breast cancer is still significant, as there are approximately 125 new cases of breast cancer and about 22 deaths per 100,000 U.S. women each year [1]. The USPSTF recommends biennial screening mammography for women aged 50 to 74 years [1]. Although other societies recommend screening from age 40 , studies have shown that the cumulative rates of false-positive mammography results are higher with annual rather than biennial screening (mammography, 61\% vs. $42 \%$ ), for women with heterogeneously or extremely dense breasts, and those aged 40 to 49 years [11]. Our study revealed a significant adherence to the USPSTF screening guidelines for breast cancer as $93 \%$ of mammograms ordered by resident physicians were performed and done according to the guidelines.

According to the Centers for Disease Control and Prevention (CDC), national screening rates for mammograms were 67.2\% with 17.3 million tests performed in 2016 [12]. Resident screening rates were greater than the national average according to the National Cancer Institute which showed that in $2015,71.6 \%$ of women aged $50-74$ years had a mammogram within the past two years [8]. At the state level, our screening rates were higher than that of New Jersey which was $78.2 \%$ which in itself is below the "Healthy People 2020" screening target of 81.1\% [13,14].

One possible reason for the high resident adherence to the screening guidelines is that at each female clinic encounter, residents are required to document patients' most recent screening mammogram for females aged 50-74 years which helps to ensure that mammography screening testing is addressed at each visit. In addition, we were able to access the results for prior mammograms (if applicable) via our EMR to help improve the validity. Although only $6.97 \%$ of the mammograms performed were done so inappropriately, this may be due to residents not adhering to the screening intervals, according to the USPSTF guidelines. Screening guidelines from other societies may have been used inadvertently at times, such as the American Cancer Society, which recommends annual mammograms from age 45 [15].

The USPSTF recommends screening for osteoporosis in women aged 65 years or older [3,16]. DXA scan is the gold standard for diagnosing osteoporosis and also as a guide for deciding which patients to treat. Osteoporosis imparts a significant cost burden, with direct medical costs in the United States estimated at \$12-20.3 billion dollars [17,18]. National DXA screening rates according to the CDC were $24.5 \%$ with a sample size of 5.8 million scans $[19,20]$. One study which looked at a cohort of $1,638,454$ female patients, screening rates were very low; $26.5 \%$, and $12.8 \%$ among women aged 65-79, and 80+ years, respectively [10]. Rates in a much smaller retrospective cohort study which sampled 50,995 women found that the incidence of DXA screening was $57.8 \%$ for women aged $65-74$, and $42.7 \%$ for women aged $\geqslant 75$ years [21].

Results from our study show that 107 DXA scans were performed with 88.7\% (95 of 107) of them being ordered appropriately according to the USPSTF recommendations. Although this is significantly higher than the screening rates from the two previously mentioned studies, $11.2 \%$ of the DXA scans ordered were done inappropriately. In addition, 10.1\% of DXA scans, which were not ordered, were done inappropriately as they should have been ordered, according to the guidelines. This is an area for improvement with regards to resident knowledge and adherence to the USPSTF guidelines for osteoporosis screening as fractures cause premature mortality, loss of independence and function and a reduced quality of life. One additional potential reason for the increased lack of our resident adherence to osteoporosis screening compared to mammograms and pap tests is the lack of a clearly defined interval for repeating DXA scans. According to the USPSTF, a lack of evidence exists about optimal intervals for repeated screening and whether repeated screening is necessary in a woman with normal bone mineral density (BMD) [22].

There are several limitations of our study such as the small sample size of our cohort of patients 
and the single center design. In addition, screening pap tests were done at an external outpatient office and we could not retrieve the results via our EMR. As a result, we did not include these results in our analysis.

Even with these limitations, our experience can provide a template to increase screening rates in the general population. Our recommendation as a single center based on our experience is for strong resident mentorship and close faculty monitoring. In addition, limiting the patient load for each resident on their respective clinic sessions allows residents to dedicate and spend more time reviewing the heath care maintenance aspect of each patient encounter. Also, with our significant Hispanic population an in-person Spanish translator was added in December 2016, who is medically certified for translation services. This has allowed for better and more effective physician to patient communication regarding the importance of screening for mammograms, pap tests and DXA scans.

Where possible, a certified inpatient language translator can be a very beneficial addition, which can minimize some of the inherent limitations of phone translation. This has improved patient compliance and allowed for an improved patient to physician interaction. Lastly, one of the most important factors which improves screening rates is the continuity of care provided in the program by the residents and the attending physicians. Our clinic was divided into various teams, each with their respective patients which allows residents and attending physicians to closely monitor all of the respective screening tests for their assigned patients.

\section{Conclusions}

Resident physician adherence to the United States Preventive Services Task Force screening guidelines for breast cancer and osteoporosis was high compared to the national and state screening rates. Implementation of an educational protocol consisting of strong faculty mentorship, continuity of care between residents, attending physicians and patients, the addition of an in-person medically certified Spanish language translator, as well as managing resident patient load so as to allow for a thorough patient encounter, can help to overcome some of the boundaries that individuals from low socioeconomic status may face.

\section{Additional Information Disclosures}

Human subjects: Consent was obtained by all participants in this study. St. Francis Medical Center Institutional Review Board issued approval SF\#17-008. This project was approved by St. Francis Medical Center Institutional Review Board. Animal subjects: All authors have confirmed that this study did not involve animal subjects or tissue. Conflicts of interest: In compliance with the ICMJE uniform disclosure form, all authors declare the following: Payment/services info: All authors have declared that no financial support was received from any organization for the submitted work. Financial relationships: All authors have declared that they have no financial relationships at present or within the previous three years with any organizations that might have an interest in the submitted work. Other relationships: All authors have declared that there are no other relationships or activities that could appear to have influenced the submitted work.

\section{References}

1. Siu AL, U.S. Preventive Services Task Force: Screening for breast cancer: U.S. Preventive Services Task Force recommendation statement. Ann Intern Med. 2016, 164:279-296. 10.7326/M15-2886

2. Moyer VA, U.S. Preventive Services Task Force: Screening for cervical cancer: U.S. Preventive Services Task Force recommendation statement. Ann Intern Med. 2012, 156:880-891.

10.7326/0003-4819-156-12-201206190-00424

3. U.S. Preventive Services Task Force: Screening for osteoporosis: U.S. Preventive Services Task Force recommendation statement. Ann Intern Med. 2011, 154:356-364. 10.7326/0003-4819-1545-201103010-00307 
4. Palonen KP, Allison JJ, Heudebert GR, Willett LL, Kiefe CI, Wall TC, Houston TK: Measuring resident physicians' performance of preventive care. J Gen Intern Med. 2006, 21:226-230. 10.1111/j.1525-1497.2006.00338.x

5. Brooks DJ: Differences in patient screening mammography rates associated with internist gender and level of training and change following the 2009 U.S. Preventive Services Task Force guidelines. J Natl Compr Canc Netw. 2016, 14:749-753.

6. Corbelli J, Borrero S, Bonnema R, et al.: Physician adherence to U.S. Preventive Services Task Force mammography guidelines. Women's Heal Issues. 2014, 24:313-319. 10.1016/j.whi.2014.03.003

7. Teoh DG, Marriott AE, Isaksson Vogel R, Marriott RT, Lais CW, Downs LS Jr, Kulasingam SL: Adherence to the 2012 national cervical cancer screening guidelines: a pilot study . Am J Obs Gynecol. 2015, 212:1-9. 10.1016/j.ajog.2014.06.057

8. Breast cancer screening. (2018). Accessed: April 15, 2018: https://progressreport.cancer.gov/detection/breast_cancer.

9. Roland KB, Benard VB, Soman A, Been N, Kepka D, Saraiya M: Cervical cancer screening among young adult women in the United States. Cancer Epidemiol Biomarkers Prev. 2013, 22:580-588. 10.1158/1055-9965.EPI-12-1266

10. Gillespie CW, Morin PE: Trends and disparities in osteoporosis screening among women in the United States, 2008-2014. Am J Med. 2017, 130:306-316. 10.1016/J.AMJMED.2016.10.018

11. Nelson HD, Pappas M, Cantor A, Griffin J, Daeges M, Humphrey L: Harms of breast cancer screening: systematic review to update the 2009 U.S. Preventive Services Task Force recommendation. Ann Intern Med. 2016, 164:256-267. 10.7326/M15-0970

12. Ashman JJ, Rui P, Okeyode T: Characteristics of office-based physician visits, 2014. NCHS Data Brief. 2017, 1-8.

13. C-17 data details. (2019). Accessed: July 3, 2019: https://www.healthypeople.gov/node/4055/data_details.

14. Screening and risk factors table. (2019). Accessed: July 3, 2019: https://statecancerprofiles.cancer.gov/risk/index.php? topic $=$ women \&risk=v05 \&race=00 \& type $=$ risk \&sortVariableName $=$ default....

15. Oeffinger KC, Fontham ET, Etzioni R, et al.: Breast cancer screening for women at average risk: 2015 guideline update from the American Cancer Society. JAMA. 2015, 314:1599-1614. 10.1001/jama.2015.12783

16. Final update summary: osteoporosis screening - US Preventive Services Task Force . (2018). Accessed: April 15, 2018:

https://www.uspreventiveservicestaskforce.org/Page/Document/UpdateSummaryFinal/osteoporosis screening.

17. Burge R, Dawson-Hughes B, Solomon DH, Wong JB, King A, Tosteson A: Incidence and economic burden of osteoporosis-related fractures in the United States, 2005-2025. J Bone Min Res. 2007, 22:465-475. 10.1359/jbmr.061113

18. U.S. Department of Health and Human Services: Bone Health and Osteoporosis: A Report of the Surgeon General. Office of the Surgeon General (US), Rockville, MD; 2004.

19. Looker AC, Frenk SM: Percentage of Adults Aged 65 and Over with Osteoporosis or Low Bone Mass at the Femur Neck or Lumbar Spine: United States, 2005-2010. National Center for Health Statistics, 2019.

20. Wright NC, Looker AC, Saag KG, Curtis JR, Delzell ES, Randall S, Dawson-Hughes B: The recent prevalence of osteoporosis and low bone mass in the United States based on bone mineral density at the femoral neck or lumbar spine. J Bone Miner Res. 2014, 29:2520-2526. 10.1002/jbmr.2269

21. Amarnath ALD, Franks P, Robbins JA, Xing G, Fenton JJ: Underuse and overuse of osteoporosis screening in a regional health system: a retrospective cohort study. J Gen Intern Med. 2015, 30:1733-1740. 10.1007/s11606-015-3349-8

22. Nelson HD, Haney EM, Dana T, Bougatsos C, Chou R: Screening for osteoporosis: an update for the U.S. Preventive Services Task Force. Ann Intern Med. 2010, 153:99-111. 10.7326/0003-4819153-2-201007200-00262 